\title{
Development and internal validation of the Comprehensive ALPPS Preoperative Risk Assessment (CAPRA) score: is the patient suitable for Associating Liver Partition and Portal vein ligation for Staged hepatectomy (ALPPS)?
}

\author{
Ivan Capobianco ${ }^{1}$, Karl J. Oldhafer ${ }^{2}$, Mohammed-Hossein Fard-Aghaie ${ }^{2}$, Ricardo Robles-Campos ${ }^{3}$, \\ Roberto Brusadin ${ }^{3}$, Henrik Petrowsky ${ }^{4}$, Michael Linecker ${ }^{4}$, Arianeb Mehrabi ${ }^{5}$, Katrin Hoffmann ${ }^{5}, \mathrm{Jun} \mathrm{Li}^{6}$, \\ Asmus Heumann ${ }^{6}$, Roberto Hernandez-Alejandro ${ }^{7}$, Mauro Enrique Tun-Abraham ${ }^{8}$, Elio Jovine', \\ Matteo Serenari $^{10}$, Bergthor Bjornsson ${ }^{11}$, Per Sandström ${ }^{11}$, Ruslan Alikhanov ${ }^{12}$, Mikhail Efanov ${ }^{12}$, \\ Paolo Muiesan $^{13}$, Andrea Schlegel ${ }^{13}$, Thomas M. van Gulik ${ }^{14}$, Pim B. Olthof ${ }^{14}$, Gregor Alexander Stavrou ${ }^{15}$, \\ Lina Maria Serna-Higuita ${ }^{16}$, Alfred Königsrainer ${ }^{1}$, Silvio Nadalin ${ }^{1}$
}

${ }^{1}$ Department of General, Visceral and Transplant Surgery, University Hospital Tübingen, Tübingen, Germany; ${ }^{2}$ Department of General and Abdominal Surgery, Asklepios Hospital Barmbek, Hamburg, Germany; ${ }^{3}$ Department of Surgery, Virgen de la Arrixaca Clinic and University Hospital, Murcia, Spain; ${ }^{4}$ Swiss HPB and Transplantation Center, Department of Surgery, Zurich University Hospital, Zurich, Switzerland; ${ }^{5}$ Department of General, Visceral, and Transplantation Surgery, University of Heidelberg, Heidelberg, Germany; ${ }^{6}$ General, Visceral and Thoracic Surgery Department and Clinic, Hamburg-Eppendorf University Medical Center, Hamburg, Germany; ${ }^{7}$ Division of Transplantation and Hepatobiliary Surgery, University of Rochester, Rochester, NY, USA; ${ }^{8}$ Division of HPB Surgery and Liver Transplantation, Department of Surgery, London Health Sciences Centre, London, Canada; ${ }^{9}$ General and Emergency Surgery Unit, Azienda Ospedaliero-Universitaria di Bologna, Maggiore Hospital, Bologna, Italy; ${ }^{10}$ General Surgery and Transplantation Unit, Azienda Ospedaliero-Universitaria di Bologna, Sant'Orsola-Malpighi Hospital, Bologna, Italy; ${ }^{11}$ Department of Surgery in Linköping, and Department of Biomedical and Clinical Sciences, Linköping University, Linköping, Sweden; ${ }^{12}$ Department of Hepatopancreatobiliary Surgery, Moscow Clinical Scientific Center, Moscow, Russia; ${ }^{13}$ Liver Unit, Queen Elizabeth Hospital Birmingham, University Hospitals Birmingham NHS Foundation Trust, Birmingham, UK; ${ }^{14}$ Department of Surgery, Amsterdam UMC (Location AMC), University of Amsterdam, Amsterdam, The Netherlands; ${ }^{15}$ Department of General, Abdominal and Thoracic Surgery, Surgical Oncology, Saarbruecken General Hospital, Saarbruecken, Germany; ${ }^{16}$ Department of Clinical Epidemiology and Applied Biometry, University of Tübingen, Tübingen, Germany

Contributions: (I) Conception and design: I Capobianco, LM Serna-Higuita, S Nadalin, A Königsrainer; (II) Administrative support: I Capobianco, KJ Oldhafer, R Robles-Campos, H Petrowsky, A Mehrabi, J Li, R Hernandez-Alejandro, E Jovine, B Bjornsson, R Alikhanov, P Muiesan, TM van Gulik, GA Stavrou, LM Serna-Higuita, S Nadalin, A Königsrainer; (III) Provision of study materials or patients: I Capobianco, MH Fard-Aghaie, R Brusadin, M Linecker, K Hoffmann, A Heumann, ME Tun-Abraham, M Serenari, B Bjornsson, M Efanov, A Schlegel, PB Olthof, GA Stavrou; (IV) Collection and assembly of data: I Capobianco, ME Tun-Abraham, M Serenari, P Sandström, M Efanov, A Schlegel, PB Olthof, GA Stavrou; (V) Data analysis and interpretation: I Capobianco, KJ Oldhafer, R Robles-Campos, H Petrowsky, M Linecker, A Mehrabi, J Li, R Hernandez-Alejandro, E Jovine, B Bjornsson, R Alikhanov, P Muiesan, TM van Gulik, PB Olthof, GA Stavrou, LM Serna-Higuita, S Nadalin; (VI) Manuscript writing: All authors; (VII) Final approval of manuscript: All authors.

Correspondence to: Silvio Nadalin, MD, FEBS. Department of General, Visceral and Transplant Surgery, University Hospital Tübingen, 72076 Tübingen, Germany. Email: silvio.nadalin@med.uni-tuebingen.de.

Background: Preoperative patient selection in Associating Liver Partition and Portal vein ligation for Staged hepatectomy (ALPPS) is not always reliable with currently available scores, particularly in patients with primary liver tumor. This study aims to (I) to determine whether comorbidities and patients characteristics are a risk factor in ALPPS and (II) to create a score predicting 90-day mortality preoperatively. Methods: Thirteen high-volume centers participated in this retrospective multicentric study. A risk analysis based on patient characteristics, underlying disease and procedure type was performed to identify risk factors and model the Comprehensive ALPPS Preoperative Risk Assessment (CAPRA) score. A nonparametric 
receiver operating characteristic analysis was performed to estimate the predictive ability of our score against the Charlson Comorbidity Index (CCI), the age-adjusted CCI (aCCI), the ALPPS risk score before Stage 1 (ALPPS-RS1) and Stage 2 (ALPPS-RS2). The model was internally validated applying bootstrapping.

Results: A total of 451 patients were included. Mortality was $14.4 \%$. The CAPRA score is calculated based on the following formula: $(0.1 \times$ age $)-(2 \times$ BSA $)+1$ (in the presence of primary liver tumor $)+1$ (in the presence of severe cardiovascular disease) +2 (in the presence of moderate or severe diabetes) +2 (in the presence of renal disease) +2 (if classic ALPPS is planned). The predictive ability was 0.837 for the CAPRA score, 0.443 for CCI, 0.519 for aCCI, 0.693 for ALPPS-RS1 and 0.807 for ALPPS-RS2. After 1,000 cycles of bootstrapping the $\mathrm{C}$ statistic was 0.793 . The accuracy plot revealed a cut-off for optimal prediction of postoperative mortality of 4.70 .

Conclusions: Comorbidities play an important role in ALPPS and should be carefully considered when planning the procedure. By assessing the patient's preoperative condition in relation to ALPPS, the CAPRA score has a very good ability to predict postoperative mortality.

Keywords: Associating Liver Partition and Portal vein ligation for Staged hepatectomy (ALPPS); comorbidity; mortality; prediction model; patient selection

Submitted Oct 11, 2021. Accepted for publication Dec 28, 2021.

doi: $10.21037 / \mathrm{hbsn}-21-396$

View this article at: https://dx.doi.org/10.21037/hbsn-21-396

\section{Introduction}

Since its introduction in 2007, Associating Liver Partition and Portal vein ligation for Staged hepatectomy (ALPPS) has been a promising two-stage technique that allows resection in patients with small future liver remnant (FLR) and therefore with poor outcome and treatment options.

According to the ALPPS registry (https://www.alpps. net/) more than 1,200 ALPPS procedures have been performed to date worldwide, however preoperative patient selection is not always reliable. This is particularly valid for patients with a primary liver cancer with no other therapeutic options, for whom new studies have demonstrated an excellent oncological outcome after ALPPS (1).

The main argument against ALPPS remains its high mortality, ranging from $9 \%$ to $17 \%(2-8)$. Great effort has been undertaken to identify the main risk factors for mortality after ALPPS, as well as to develop predictive mortality scores to improve patient selection $(5,6,9)$.

Linecker et al. showed that basing patient selection on younger age, shifting the indication towards colorectal liver metastasis (CRLM), developing less invasive approaches and appropriate timing of Stage 2 have reduced mortality in recent years to less than $5 \%(1,6,8,10)$. The same study also showed that the selection of patients with comorbidities such as cardiovascular and renal disease was significantly reduced over the years.

Patient comorbidities are known to be a risk factor for postoperative mortality in any surgical procedure, particularly in patients developing sepsis or posthepatectomy liver failure (11-14). However, comorbidities (taken individually or globally) are not yet recognized as a significant risk factor in the ALPPS literature $(5,9)$. Cardiovascular disease has marginally failed statistical significance in some reports (9), while the overall measurement of comorbidities, usually assessed with the Charlson Comorbidity Index (CCI) or the age-adjusted CCI (aCCI) $(15,16)$, has systematically failed as a measure of 90-day mortality predictivity in ALPPS $(5,9,17)$.

In addition, already available predictive scores of 90-day mortality, such as the validated ALPPS risk score (18) and the newly proposed risk score for CRLM (17), have been shown to have before Stage 1 only an acceptable predictivity value with a $C$ statistic of 0.64 and 0.70 , respectively.

To overcome this limitation, we conducted a multicentric retrospective study for the purpose of determining the true role of comorbidities in ALPPS and developing a preoperative risk score based on patients characteristics for predicting mortality risk related to the procedure and consequently for accurately selecting patients for the ALPPS procedure. 


\section{Aim}

The primary goal of the study was to determine whether and which comorbidity, alone or aggregated, is a risk factor for postoperative mortality after completion of ALPPS.

The secondary aim was to develop a model for preoperatively predicting postoperative mortality (defined as procedure-related mortality after Stage 1 or 90 -day mortality after Stage 2) and consequently selecting patients for the ALPPS procedure. The guidelines of the "transparent reporting of a multivariable prediction model for individual prognosis or diagnosis" (TRIPOD) statement were followed. We present the following article in accordance with the TRIPOD reporting checklist (available at https://hbsn. amegroups.com/article/view/10.21037/hbsn-21-396/rc).

\section{Methods}

\section{Study design}

A multicentric retrospective study was conducted in patients undergoing ALPPS between November 2010 and February 2019. The cases were submitted by 13 high-volume hepatobiliary centers worldwide, defined as centers with an experience of at least 20 ALPPS each. The study was conducted in accordance with the Declaration of Helsinki (as revised in 2013). The study was approved by Independent Ethics Committee (IEC) of Tübingen University Hospital (No. 030/2019A) and informed consent was taken from all individual participants.

\section{Inclusion and exclusion criteria}

All submitted cases were critically reviewed for inclusion in the study. Inclusion criteria were any type of ALPPS without exception for surgical technical variation, extension of resection or type of tumor. Exclusion criteria were age under 18 years, aborted procedure after Stage 1 or Interstage longer than 90 days.

\section{Definitions of variables and outcome measures}

Patient characteristics such as age, weight, height, body mass index (BMI), body surface area (BSA) according to the Mosteller formula (19) were recorded. The standard liver volume (SLV) was calculated with Vauthey's formula (20). The type of tumor was recorded and then classified as primary or secondary. The comorbidities were defined as done by Charlson in her original manuscript (16) and summarized in Table S1.

The following scores were evaluated: (I) the CCI and (II) the aCCI were calculated as known global measures of comorbidity condition $(15,16)$. (III) The already validated ALPPS risk score before Stage 1 (ALPPS-RS1) and (IV) before Stage 2 (ALPPS-RS2) were also evaluated as the only known predictive score for 90-day mortality following ALPPS that can be applied to any type of tumor $(9,18)$.

Outcomes such as severe complications (defined as Grade 3b or higher according to the Dindo-Clavien classification) (21), length of hospital stay and postoperative mortality (defined as procedure-related mortality after Stage 1 or 90-day mortality after Stage 2) were recorded. Cancer-related mortality was not considered in the study.

To exclude a learning curve effect, we also evaluated possible differences between the first 10 patients at each center and the others.

\section{Surgical approach}

Classic ALPPS was defined as an open approach with surgical portal occlusion (ligature or dissection) and complete parenchymal transection $(7,22,23)$, while partial ALPPS was defined as incomplete parenchymal transection, regardless of the portal occlusion technique $(24,25)$. Tourniquet ALPPS was defined as described by Robles et al. (26) and was considered an incomplete parenchymal transection. Radiofrequency-assisted ALPPS (RALPPS) was defined as parenchymal transection by means of radiofrequency ablation with partial parenchyma partition (27). Hybrid ALPPS was defined as intra- or perioperative embolization of the portal vein (PVE) associated with complete or partial surgical transection $(28,29)$, while PVE associated with other transection methods (e.g., tourniquet ALPPS or RALPPS) was reported separately. Any laparoscopic or other minimally invasive approach was also recorded.

Extended hepatectomy was defined according to Brisbane's classification as the resection of more than four segments (30).

\section{Objectives and endpoints}

The primary endpoint of this study was the postoperative mortality (defined as procedure-related mortality after Stage 1 or 90-day mortality after Stage 2). 


\section{Statistical methods}

Descriptive statistics were used to describe baseline characteristics. Categorical variables are reported as an absolute and relative frequencies number. Continuous variables were expressed as mean and standard deviation (SD) for normal distribution, otherwise as median and interquartile range (IQR). Data normality was tested by visual inspection (histogram, boxplot and Q-Q plot), Skewness (between -1 and +1 ) and kurtosis $(-2$ and +2 ).

Bivariate analysis was carried out to compare patients alive or not alive at 90 days. Qualitative variables were compared with the $\chi^{2}$ test or Fisher's exact test, as appropriate. Independent samples $t$-test was used to compare continuous variables that were normally distributed, while Mann-Whitney $U$ test was used to evaluated variables with non-normal distribution.

All statistical analyses were performed with IBM SPSS Statistics, version 25 (IBM Corporation, Armonk, NY, USA).

\section{Model development}

Binary logistic regression was performed to assess associations with mortality at 90 days. Candidate variables for the multivariate model were selected between variables with a data completion rate of at least $80 \%$, based on clinical knowledge and those variables with a $\mathrm{P}$ value less than 0.1 in the univariate analysis. Spearman's correlation coefficient was used to detect the collinearity of the variables in the regression model. The strength of clinical variables to predict prognosis was assessed based on odds ratios $(\mathrm{OR}), 95 \%$ confidence intervals $(\mathrm{CI})$. $\mathrm{P}$ values equal or less than 0.05 was considered statistically significant and these variables were considered in the calculation of the final model.

To maximize predictivity and minimize the information lost, continuous variables were preferred over cut-off variables (31). In addition, to avoid inclusion or exclusion of predictors by chance, grouping of less prevalent comorbidities based on clinical and statistical usefulness was considered.

The 1-in-10 rule (one covariate every ten observations) was used to decide which variables to include in the model and confirmed with the Riley equation with a confidence interval of $95(32,33)$.

The final predictors in the model were selected using forward selection based on the lowest Akaike information criterion (AIC), and these were given a weight based on the relative size of were given a weight based on the relative size of logistic regression coefficients.

Calibration was evaluated using the HosmerLemershow test and calibration slope. Performance of the models developed was assess using the area under the receiver operating curve (AUC). A C statistic below 0.70 was considered poor discrimination, above 0.70 acceptable, while greater than 0.80 it was considered a good discrimination. DeLong's test was used to test whether the AUCs of the models are statistically different (34).

\section{Internal validation}

To account for model optimism in terms of overfitting, the new score developed was internally validated applying bootstrapping. We repeated the modeling process in 1,000 bootstrap samples. The result of the validations was assessed by ROC curve and by estimating sensitivity and specificity of the clinical prediction rule.

Moreover the model was tested on selected subgroups of patients and the ROC curve was calculated.

\section{Results}

\section{Patient characteristics}

A total of 482 patients who underwent ALPPS between 2010 and 2019 in 13 centers worldwide were submitted and screened for inclusion in the study. Each center had a median (IQR) experience of 34 ALPPS (25-51.5) procedures. Thirty-one $(6.4 \%)$ patients were excluded due to various exclusion criteria such as incomplete procedure ( 25 cases; $5.1 \%$ ), Interstage longer than 90 days (range, $100-530 ; 11$ cases; $2.3 \%$ ), age under 18 years (2 cases; $0.41 \%)$. Seven $(1.45 \%)$ patients presented more than one exclusion criterion. Finally, 451 patients were included in the study.

The characteristics of the patient cohort and the completeness of the data are shown in Table 1 .

Overall mean age was $61.6 \pm 11.4$ years.

There were 296 (65.6\%) secondary liver tumors [(267 CRLM; 59.2\%) and 29 other metastatic cancer (6.4\%)] and $155(34.4 \%)$ primary tumors [36 HCC (8.0\%), 109 biliary tumors $(24.2 \%)$ - of which 50 were intrahepatic CCA (11.1\%), 50 perihilar CCA (11.1\%), and 9 gallbladder cancer (2.0\%) - and 10 other non-HCC/non-CCA tumors (2.2\%)].

Comorbidities were seen in $259(57.4 \%)$ patients, 141 $(31.3 \%)$ of them presented more than one comorbidity. 
Table 1 Baseline characteristics and comparison of patients who experienced postoperative mortality or not

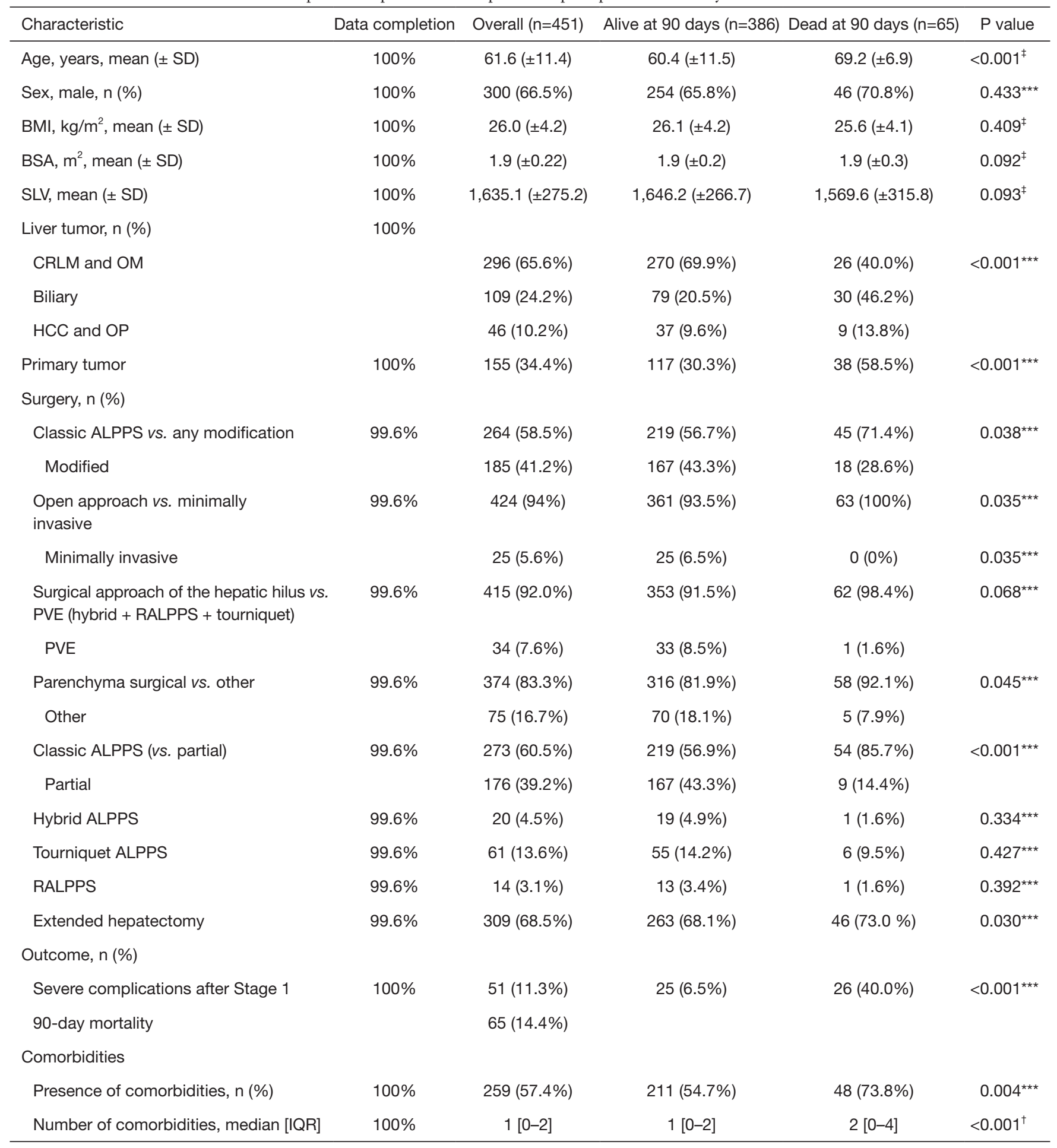

Table 1 (continued) 
Table 1 (continued)

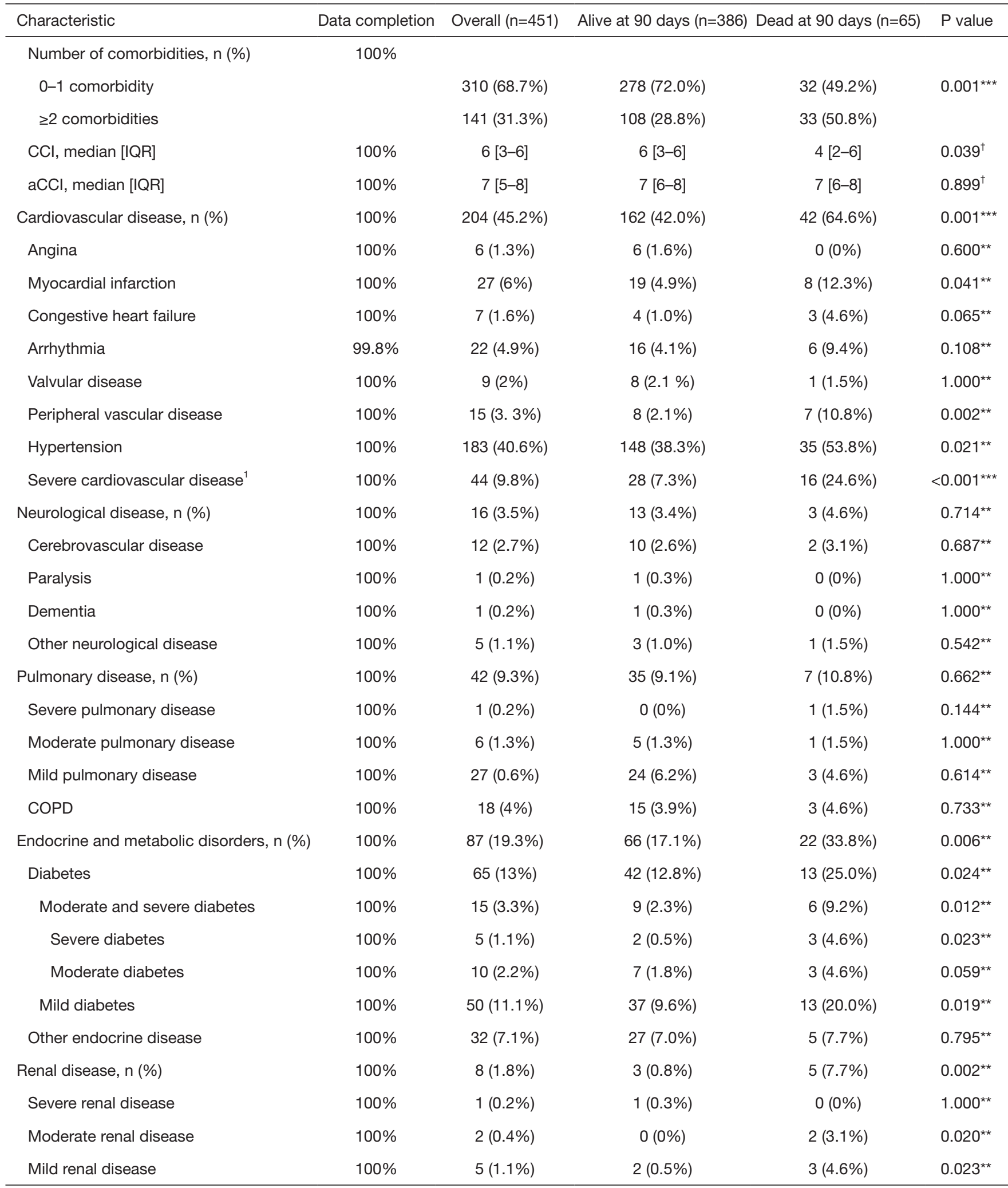

Table 1 (continued) 
Table 1 (continued)

\begin{tabular}{|c|c|c|c|c|c|}
\hline Characteristic & Data completion & Overall $(n=451)$ & Alive at 90 days $(n=386)$ & Dead at 90 days $(n=65)$ & $P$ value \\
\hline Severe liver disease & $100 \%$ & - & $0(0 \%)$ & $0(0 \%)$ & - \\
\hline Moderate liver disease & $100 \%$ & $8(1.8 \%)$ & $7(1.8 \%)$ & $1(1.5 \%)$ & $1.000^{* \star}$ \\
\hline Mild liver disease & $100 \%$ & $14(3.1 \%)$ & $12(3.1 \%)$ & $2(3.1 \%)$ & $1.000^{* *}$ \\
\hline Inflammatory bowel disease & $100 \%$ & $3(0.7 \%)$ & $1(0.3 \%)$ & $2(3.1 \%)$ & $0.056^{\star *}$ \\
\hline Peptic ulcer & $100 \%$ & $12(2.7 \%)$ & $10(2.6 \%)$ & $2(3.1 \%)$ & $0.687^{\star \star}$ \\
\hline Gastrointestinal bleeding & $100 \%$ & $1(0.2 \%)$ & $0(0 \%)$ & $1(1.5 \%)$ & $0.144^{* *}$ \\
\hline $\begin{array}{l}\text { Immunological or hematological } \\
\text { disease, } \mathrm{n}(\%)\end{array}$ & $100 \%$ & $16(3.5 \%)$ & $12(3.1 \%)$ & $4(6.2 \%)$ & $0.266^{\star \star}$ \\
\hline Leukemia & $100 \%$ & $1(0.2 \%)$ & $1(0.3 \%)$ & $0(0 \%)$ & $1.000^{\star *}$ \\
\hline Rheumatologic disease & $100 \%$ & $11(2.4 \%)$ & $7(1.8 \%)$ & $4(6.2 \%)$ & $0.059^{* *}$ \\
\hline Coagulopathy & $100 \%$ & $1(0.2 \%)$ & $1(0.3 \%)$ & $0(0 \%)$ & $1.000^{\star \star}$ \\
\hline Connective tissue disease, $\mathrm{n}(\%)$ & $34.6 \%$ & $6(4.5 \%)$ & $4(3.4 \%)$ & $2(11.8 \%)$ & $0.167^{\star \star}$ \\
\hline Any tumor, n (\%) & $100 \%$ & $11(2.4 \%)$ & $10(2.6 \%)$ & $1(1.5 \%)$ & $1.000^{\star *}$ \\
\hline Non-metastatic solid tumor & $100 \%$ & $11(2.4 \%)$ & $10(2.6 \%)$ & $1(1.5 \%)$ & $1.000^{\star *}$ \\
\hline Metastatic solid tumor & $100 \%$ & $0(0 \%)$ & $0(0 \%)$ & $0(0 \%)$ & - \\
\hline
\end{tabular}

The comorbidities are defined as done by Charlson in her original manuscript and summarized in Table S1. \% is referred to columns Overall, Alive or Dead at 90 days respectively. ${ }^{\ddagger}$, independent samples $t$-test; ${ }^{* \star}$, Chi square test; ${ }^{\dagger}$, Mann-Whitney test; ${ }^{* \star}$, Fischer's exact test; ${ }^{1}$, severe cardiovascular disease: any of congestive heart disease, myocardial infarction or peripheral vascular disease. SD, standard deviation; BMI, body mass index; BSA, body surface area according to the Mosteller formula; SLV, standard liver volume according to the Vauthey formula; CRLM, colorectal liver metastasis; OM, other metastatic liver tumor; HCC, hepatocellular carcinoma; OP, other primary liver tumor; ALPPS, Associating Liver Partition and Portal vein ligation for Staged hepatectomy; RALPPS, radiofrequency-assisted ALPPS; PVE, portal vein embolization; IQR, interquartile range; aCCI, age-adjusted Charlson Comorbidity Index; $\mathrm{CCI}$, Charlson Comorbidity Index.

Median CCI and aCCI were 6 (IQR, 3-6) and 7 (IQR, 5-8), respectively.

Mean ALPPS-RS1 and ALPPS-RS2 were $1.5( \pm 1.6)$ and $3.8( \pm 1.5)$, respectively, reflecting a 90 -day mortality risk from $2.7 \%$ to $15 \%$ for Stage 1 and up to $20 \%$ for Stage 2, based on data from the original manuscript (9).

In the learning curve group, we included 117 patients, 67 of which $(57.3 \%)$ presented at least one comorbidity, 17 patients $(14.5 \%)$ had 90 -days mortality. The postlearning group included 334 patients, 192 of which $(57.5 \%)$ presented at least one comorbidity, 48 patients (14.4\%) had 90-days mortality.

\section{Surgical characteristics}

Median Interstage interval between Stage 1 and Stage 2 was 13 (IQR, 9-17) days.

Operative characteristics were provided for 449 (99.5\%) patients and are shown in Table 1; 264 (58.8\%) patients underwent classic ALPPS, while 185 (41.2\%) underwent 
any technical modification in Stage 1. Among these procedures, we recorded 176 partial ALPPS (39.2\%), 61 tourniquet ALPPS (13.5\%), 34 cases of PVE ALPPS (7.6\%)—of which 20 were hybrid ALPPS with complete or partial surgical parenchymal transection $(4.5 \%)-25$ laparoscopic or other minimally invasive approach (5.6\%) and 14 RALPPS (3.1\%).

Right trisectionectomy was performed in 300 cases $(66.8 \%)$, right hemihepatectomy in 137 (30.5\%), monosegmental ALPPS in 5 (1.1\%), left hemihepatectomy in $3(0.7 \%)$, left trisectionectomy in $2(0.4 \%)$, extended left hepatectomy with FLR corresponding to segment 5-6 in $2(0.4 \%)$ cases. A total of $309(68.8 \%)$ patients required an extended hepatectomy.

\section{Overall outcome}

\section{Morbidity}

The rate of severe complications ( $\geq 3 \mathrm{~b}$ ) following Stages 1 and 2 was $11.3 \%$ (51 events) and 28.2\% (127 events), respectively. Mean hospital stay following Stage 2 was 14 days $( \pm 7.4)$.

The incidence of severe complications was higher but not significantly different in patients with at least one comorbidity, both in the Interstage $(13.5 \%$ in patients with comorbidities vs. $8.3 \%$ in patients without, $\mathrm{P}=0.099$ ) and after Stage 2 (30.4\% in patients with comorbidities vs. $26.3 \%$ in patients without, $\mathrm{P}=0.396$ ).

\section{Mortality}

Sixty-five (14.4\%) patients died in the postoperative course [10 (2.2\%) after Stage 1 and 55 (12.2\%) within 90 days after Stage 2].

The differences between patients who experienced postoperative mortality or not are provided in Table 1.

Reported mortality for secondary tumors was $9.1 \%$ [27/296 cases, of which 25 were CRLM (CRLM mortality: $9.4 \%)]$ and for primary tumors $24.5 \%$ [38/155 cases, of which 30 were CCA (CCA mortality: $27.5 \%$ ) and 7 HCC (HCC mortality: 19.4\%)].

Patients who deceased were older (69.2 vs. 60.4 mean years of age, $\mathrm{P}<0.001)$, had a higher prevalence of primary tumors $(58.5 \%$ vs. $30.3 \%, \mathrm{P}<0.001)$ and comorbidities (73.8\% vs. $54.7 \%, \mathrm{P}=0.004)$, particularly cardiovascular (64.6\% vs. $42.0 \%, \mathrm{P}=0.001$ ), renal disease $(7.7 \%$ vs. $0.8 \%$, $\mathrm{P}=0.002)$ and diabetes (25.0\% vs. $12.8 \%, \mathrm{P}=0.024)$. In addition, patients with postoperative mortality were more likely to have undergone the classic ALPPS procedure
(71.4\% vs. $56.7 \%, \mathrm{P}=0.038)$.

\section{Detectable effect}

The present analysis was carried out on a fixed sample of 451 patients. Power was calculated ex-post based on the risk of postoperative mortality. Using the software PASS 2020, the group sample size of 451 achieve an $88 \%$ power to detect and OR of 2.2 if the proportion of event is 0.14 .

\section{Prediction model of postoperative mortality}

For the purpose of preoperative risk assessment, a univariate analysis was performed using only patient covariates and surgical variables (see also Table S2).

The percentage of missing variables included in the model was $2.4 \%$, therefore the analysis was done using complete cases analysis.

According to the 1-in-10 rule and in order to prevent overfitting of the model, for the 65 mortality events we aimed to include six to seven variables in the final model (32). Riley's equation with seven variables, an event proportion of 0.14 , and a 95 confidence interval confirm that the power of the model was sufficient, as the required sample size should be at least 230 patients (33).

The following seven variables were identified as predictors of postoperative mortality: (I) age, (II) BSA, (III) primary liver tumor, (IV) severe cardiovascular disease (defined as the presence of any congestive heart disease, myocardial infarction or peripheral vascular disease), (V) renal disease, (VI) mild or severe diabetes and (VII) classic ALPPS (in terms of complete parenchymal transection) (Table 2).

Inflammatory bowel disease was discarded from the final model because of its low prevalence and the resulting extremely wide CI 95\% (OR $=25.497$; 95\% CI: 0.829 784.310).

From the above data we developed a 90-day mortality risk score that we named Comprehensive ALPPS Preoperative Risk Assessment (CAPRA) score (see Table 2), based on the following formula: $(0.1 \times$ age $)-(2 \times$ BSA $)$ +1 (in the presence of primary liver tumor) +1 (in the presence of severe cardiovascular disease) +2 (in the presence of moderate or severe DM) +2 (in the presence of renal disease) + 2(if classic ALPPS is planned).

The exact probability of 90-day mortality for each point can be calculated according to the following formula: Odds/ $(1+O d d s)$, where Odds $=\operatorname{EXP}(-5.879+0.821 \times C A P R A$ 
Table 2 Multivariate analysis for mortality and CAPRA score modeling

\begin{tabular}{|c|c|c|c|c|c|}
\hline Variable & Regression coefficient & OR & $95 \% \mathrm{Cl}$ & $P$ value & CAPRA score \\
\hline $\mathrm{BSA}^{\dagger}$ & -1.788 & 0.167 & $0.040-0.708$ & 0.015 & $-2 * B S A$ \\
\hline Primary liver tumor & 0.936 & 2.550 & $1.385-4.695$ & 0.003 & +1 \\
\hline Severe cardiovascular disease & 0.859 & 2.360 & $1.033-5.394$ & 0.042 & +1 \\
\hline Renal disease & 1.779 & 5.922 & $1.146-30.605$ & 0.034 & +2 \\
\hline Classic ALPPS & 1.703 & 5.491 & $2.369-12.729$ & $<0.001$ & +2 \\
\hline
\end{tabular}

The table shows the respective logistic regression coefficients and OR with $95 \% \mathrm{Cl}$ of the final variables. The identified predictors were incorporated in a function based on the relative size of the logistic regression coefficients that we call the CAPRA score: $(0.1 \times$ age $)-(2 \times$ $\mathrm{BSA})+1$ (in the presence of primary liver tumor) +1 (in the presence of severe cardiovascular disease) +2 (in the presence of moderate or severe DM) +2 (in the presence of renal disease) +2 (if classic ALPPS is planned). ${ }^{\dagger}$, positive prognostic factors. CAPRA, Comprehensive ALPPS Preoperative Risk Assessment; OR, odds ratios; Cl, confidence intervals; BSA, body surface area according to the Mosteller formula; DM, diabetes mellitus; ALPPS, Associating Liver Partition and Portal vein ligation for Staged hepatectomy.

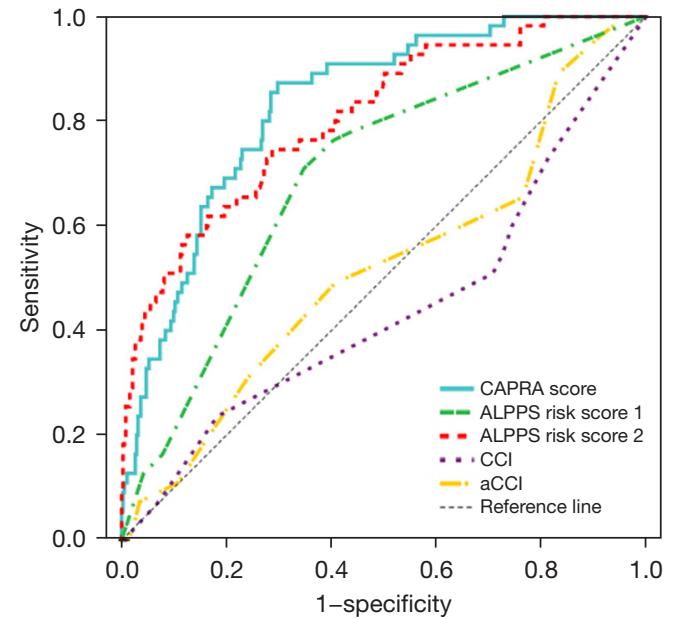

Figure 1 ROC curve for CAPRA score in comparison with ALPPS risk score 1, ALPPS risk score 2, CCI and aCCI. CAPRA, Comprehensive ALPPS Preoperative Risk Assessment; ALPPS, Associating Liver Partition and Portal vein ligation for Staged hepatectomy; aCCI, age-adjusted Charlson Comorbidity Index; CCI, Charlson Comorbidity Index.

score).

Using all seven independent predictors, the model showed good calibration with a Hosmer-Lemeshow $\chi^{2}$ of 4.8 and a $\mathrm{P}$ value of 0.779 . The ROC curves for the various predictors are shown in Figure 1.

Predictivity was good for the CAPRA score (C statistic: 0.837; 95\% CI: 0.789-0.885) and for the ALPPS-RS2
(C statistic: 0.807; 95\% CI: 0.744-0.870). Predictivity was poor for ALPPS-RS1 (C statistic: 0.693; 95\% CI: $0.621-$ 0.766), aCCI (C statistic: 0.519; 95\% CI: 0.434-0.604) and CCI (C statistic: 0.443; 95\% CI: 0.354-0.531) (Figure 1).

The difference between the $\mathrm{C}$ statistics was statistically significant for CAPRA score vs. the ALPPS-RS1 $(\mathrm{P}<0.001)$ but not $v$ s. the ALPPS-RS2 ( $\mathrm{P}=0.4576)$.

The risk probability for each interval of the CAPRA score is summarized in Figure 2. The accuracy plot revealed a cut-off for optimal prediction of postoperative mortality of 4.70 (sensitivity 0.857 and specificity 0.714 ), corresponding to a risk of $12 \%$, with an accuracy of $73 \%$.

After 1,000 cycles of bootstrapping the $\mathrm{C}$ statistic was 0.793 , and the average optimism was 0.01 (95\% CI: $0.001-$ 0.002 ) suggesting minimal overfitting.

The C statistic calculated on selected subgroups showed overall good discrimination. It was 0.814 for primary liver tumors (95\% CI: 0.741-0.887), 0.818 for secondary liver tumors (95\% CI: $0.745-0.891$ ), 0.789 for classic-ALPPS (95\% CI: 0.729-0.850), 0.829 for partial-ALPPS (95\% CI: $0.704-0.955), 0.844$ if more than 4 segments were resected (95\% CI: 0.789-0.899), 0.804 in the learning group (95\% CI: $0.718-0.890)$ vs. 0.845 in the post-learning group $(95 \%$ CI: 0.788-0.903).

\section{Discussion}

The high postoperative mortality rate is the main concern related to ALPPS (2-8). While selection criteria based 


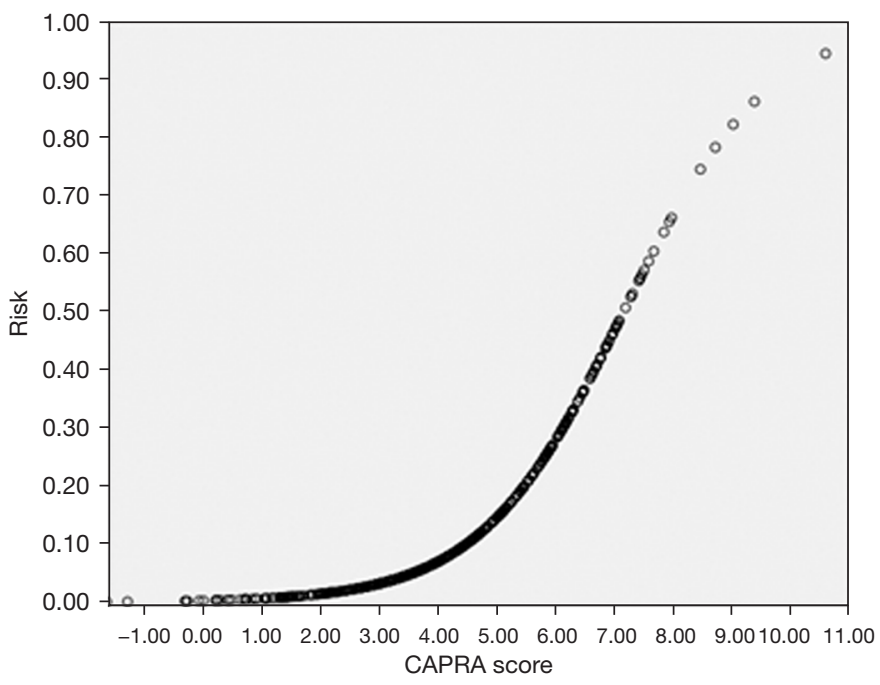

\begin{tabular}{cc} 
CAPRA score & $\begin{array}{c}\text { Probability of } \\
90 \text { d mortality }\end{array}$ \\
\hline$\leq 4$ & $\leq 7 \%$ \\
\hline 4.5 & $10 \%$ \\
\hline 5 & $15 \%$ \\
5.5 & $20 \%$ \\
6 & $25 \%$ \\
\hline 6.5 & $37 \%$ \\
\hline 7 & $45 \%$ \\
\hline 7.5 & $57 \%$ \\
\hline 8 & $65 \%$ \\
\hline$\geq 9$ & $\geq 80 \%$ \\
\hline
\end{tabular}

Figure 2 Distribution and probability of 90-day mortality for each interval of the CAPRA score. The exact probability can be calculated for each point according to $O d d s /(1+O d d s)$, where $O d d s=E X P(-5.879+0.821 \times C A P R A$ score $)$. The difference in probability growth for the same range is explained by the logarithmic nature of the model. CAPRA, Comprehensive ALPPS Preoperative Risk Assessment.

on liver volume, patient age and tumor type have already been widely discussed $(5,6,8,9,35)$, less is known about the interaction between the patient's comorbidity status and outcome.

This study shows for the first time that it is mandatory to consider the comorbid status in patients undergoing ALPPS and confirms that careful selection based on patient characteristics and condition can further prevent mortality following ALPPS, particularly in patients with primary tumor, such as HCC and CCA. Cardiovascular diseases, diabetes and renal disease are the most prevalent comorbidities in patients with fatal outcome.

In light of this, the main aim of this study was to develop a risk score that can already be applied in the patient selection stage, namely before performing ALPPS, to estimate the probability of postoperative mortality. The study identified the following seven variables as independent predictors of postoperative mortality following ALPPS: (I) age, (II) BSA, (III) primary liver tumors, comorbidities such as (IV) severe cardiovascular disease, (V) mild or severe diabetes and (VI) renal disease and (VII) classic ALPPS.

Advanced age has been repeatedly indicated as a risk factor for ALPPS $(2,5,8,29,36)$. Various cut-offs over the age of 60 have been arbitrarily proposed and analyzed $(2,5)$. The study supporting the ALPPS risk score, which was based on an accuracy analysis, indicated age 67 as the optimal cut-off for predicting 90-day mortality (9). In the present study, however, we preferred to leave age as a continuous variable in order to not lose relevant statistical information (31).

Interestingly, BSA has shown strong predictivity in multivariate analysis. BSA is related to estimated total and future liver volume $(20,35,37,38)$ and, not without criticism, is widely used as a reflection of hepatic and renal metabolism to calculate the appropriate dose of drugs $(39,40)$. However, since the reason for its predictive ability has yet to be determined, in this type of study (unlike the etiological study) even non-causal variables can be highly predictive of outcome (41).

This study confirms previous findings showing that patients with secondary liver tumor, particularly CRLM, experience less postoperative mortality $(5,8,9)$. The predictive ability of the primary tumor as a whole was superior to that of sub-classified tumors of different types, particularly HCC and CCA. This could perhaps be explained by the fact that primary tumors usually develop in an already diseased liver and in older patients, whereas metastasis develop in a healthy liver and in younger patients $(2,9,42)$.

Three comorbidities were included in the final model: severe cardiovascular disease (defined as any of myocardial infarction, congestive heart failure or peripheral vascular disease), moderate or severe diabetes mellitus and renal disease. We can assume that comorbidities reduce the ability to overcome complications once they have occurred, 
because for a similar number of serious complications, patients with comorbidities have significantly higher mortality.

These comorbidities, defined or classified in different ways, have already been analyzed in other studies. A higher prevalence of comorbidities has already been observed among patients with postoperative mortality $(8,9,14)$. Comorbidities, however, have so far failed to demonstrate significance in ALPPS or, as overall cardiovascular disease in the ALPPS risk score pre-Stage 1, did not increase the model's performance (9). This can be explained by the fact that the other studies included fewer fatal events and that it is plausible that observations were underreported, as they were based on registry entries and did not focus primarily on comorbidities.

Moreover, the overall measurement of comorbidities, usually assessed with the CCI or the aCCI $(15,16)$, has systematically failed as a measure of 90-day mortality predictivity in ALPPS $(5,9,17)$. We believe that this contradictory situation is mainly due to the intrinsic imbalance of the oncological state in CCI and consequently aCCI, since the points attributed to secondary tumors outweigh the points given to other comorbidities. Considering the fact that CCI and aCCI each assign to a secondary liver tumor (e.g., CRLM) four points more than to a primary liver tumor, we believe that the true comorbid condition of these patients is often wrongly overestimated by comparison to that of patients with primary liver cancer [e.g., hepatocellular carcinoma (HCC) or cholangiocarcinoma (CCA)]. Consequently, CCI and aCCI are of limited value for assessing a patient's comorbidity status and determining comorbidity as a risk factor for mortality in ALPPS.

Several technical modifications of the ALPPS procedure have been proposed in recent years to reduce associated morbidity and mortality $(43,44)$. Prospective controlled studies confirming the superiority of one technique over the other are still lacking. However, a switch to less invasive approaches has already been highlighted and associated with a decline in mortality in recent years (8). This study confirms that of all the modified techniques a partial parenchymal transection has shown overall superiority over classic ALPPS in terms of safety, thus supporting the idea that reducing operative trauma in Stage 1 improves outcome $(8,24,25,45)$. This included either an open or laparoscopic approach, PVE ALPPS, tourniquet ALPPS or RALPPS. However, it should not be forgotten that modified approaches are still in the minority and, among them, partial ALPPS is the most common, even when combined with other techniques. Moreover, as already pointed out (8), all the modifications were introduced in recent years, possibly overlapping the learning curve and hiding a bias.

Of note, since the CAPRA score is an almost static model, the only possible intervention the clinician can undertake to reduce the operative risk involves the type of procedure to be performed.

Interestingly, patients undergoing extended hepatectomy are more prevalent in the mortality group. However, this operative characteristic is still not significant in the multivariate analysis (17).

The model generated, using variables available prior to Stage 1, showed good performance in predicting postoperative mortality with a $\mathrm{C}$ statistic of 0.837 and allows good estimation of individual patient risk also after bootstrapping or subgrouping. As far as we know, the CAPRA score is much better than the already validated models that can be applied before performing ALPPS, such as the ALPPS risk score 1, and comparable to the ALPPS risk score 2 (9), which, however, requires information only available in Interstage period and therefore not suitable before Stage 1. Since the CAPRA score can quantify with good accuracy the mortality risk of the procedure, both the patient and the surgical center are supported in their decision to undergo or perform the ALPPS procedure, respectively, or, if possible, plan other examinations or evaluate alternative augmentation techniques.

The strength of this study is associated with the large sample size of 451 patients from 13 high-volume centers with almost full completion of the data set. Since we contacted the centers individually, we overwhelmed the reporting bias that can characterize registry-based studies, where negative events tend to be underreported $(9,17)$. The 65 mortality events collected are actually the largest sample reported in an ALPPS risk study, thereby strengthening the statistical power of the analysis (46). Moreover, locking the primary endpoint to 90 -day mortality rules out data interpretation or manipulation and allows this model to be compared with the others already available for ALPPS $(9,17,18)$. Finally, unlike the ALPPS risk score and the CRLM-based risk score, in the CAPRA score the type of tumor is not the decisive factor for assessing risk before Stage 1. Consequently, the mortality risk can also be better assessed in patients with primary liver tumors such as HCC and CCA, which lack treatment options but can still benefit from ALPPS (1). All this is confirmed in the internal validation and score consistency among the different 
subgroups. In particular, the CAPRA score showed good predictivity even when used during the learning curve.

On the other hand, the main limitations of the present study are its retrospective nature and the low prevalence of many comorbidities, like liver disease, in already hyperselected patients. This could lead to exclusion of some covariates by chance. For this reason, the risk in the face of any other comorbidity excluded from the model should be further assessed individually.

In addition, other liver-related factors, such as volumetry and functionality, were not included as they were not the primary focus of this study, since we wanted to determine the risk related to patient condition before Stage 1 . However, it should be noted that ALPPS is necessary only when liver quality or function is poor or volume is not adequate. Since standardized criteria for quality and functionality are still missing (47-50) and no retrospective data were available, we decided to omit these variables and focus on patient characteristics and associated procedure. Furthermore, Linecker et al. (9) found no predictive value for preoperative volumetry when assessing the ALPPS risk score. On the other hand, Huiskens et al. (17), working solely with CRLM, suggested that FLR volume to body weight ratio (FLR/BW) $<0.4$ could be predictive of 90 -day mortality and proposed PVE in such patients. It should be remembered that the proposed cut-off for primary liver resection in healthy liver is $0.5(23,51)$, and no indication of the liver quality, such as the presence of CASH, was provided in the study. In addition, this would exclude the majority of patients, as $66 \%$ of the overall patients in that study and $64 \%$ of those patients who did not experience mortality reported a FLR/BW $<0.4$. Above all, adequate FLR should be achieved before proceeding to Stage 2 rather than to the selection for Stage 1. Li et al. (1) demonstrated that insufficient FLR at Stage 2 (defined as FLR/BW <0.80) is a risk factor for severe complications and consequently for mortality. Again, the risk related to this additional information should be integrated and assessed individually.

Finally, an external validation study should be performed.

In conclusion, comorbidities play an important role and should be considered part of ALPPS planning. The CAPRA score has an excellent preoperative prediction of mortality and therefore it is an excellent tool for assessing the risk related to patient condition, once the need for ALPPS is considered, providing additional support for patient selection and procedure planning in combination with already validated scores, assessments of liver volumetry and function $(9,52)$.

\section{Acknowledgments}

Funding: None.

\section{Footnote}

Reporting Checklist: The authors have completed the TRIPOD reporting checklist. Available at https://hbsn. amegroups.com/article/view/10.21037/hbsn-21-396/rc

Data Sharing Statement: Available at https://hbsn. amegroups.com/article/view/10.21037/hbsn-21-396/dss

Conflicts of Interest: All authors have completed the ICMJE uniform disclosure form (available at https://hbsn. amegroups.com/article/view/10.21037/hbsn-21-396/coif). HP and TMvG serve as unpaid editorial board members of Hepatobiliary Surgery and Nutrition. The other authors have no conflicts of interest to declare.

Etbical Statement: The authors are accountable for all aspects of the work in ensuring that questions related to the accuracy or integrity of any part of the work are appropriately investigated and resolved. The study was conducted in accordance with the Declaration of Helsinki (as revised in 2013). The study was approved by Independent Ethics Committee (IEC) of Tübingen University Hospital (No. 030/2019A) and informed consent was taken from all individual participants.

Open Access Statement: This is an Open Access article distributed in accordance with the Creative Commons Attribution-NonCommercial-NoDerivs 4.0 International License (CC BY-NC-ND 4.0), which permits the noncommercial replication and distribution of the article with the strict proviso that no changes or edits are made and the original work is properly cited (including links to both the formal publication through the relevant DOI and the license). See: https://creativecommons.org/licenses/by-nc-nd/4.0/.

\section{References}

1. Li J, Moustafa M, Linecker M, et al. ALPPS for Locally Advanced Intrahepatic Cholangiocarcinoma: Did Aggressive Surgery Lead to the Oncological Benefit? An International Multi-center Study. Ann Surg Oncol 2020;27:1372-84.

2. Schadde E, Ardiles V, Robles-Campos R, et al. 
Early survival and safety of ALPPS: first report of the International ALPPS Registry. Ann Surg 2014;260:829-36; discussion 836-8.

3. Schadde E, Ardiles V, Slankamenac K, et al. ALPPS offers a better chance of complete resection in patients with primarily unresectable liver tumors compared with conventional-staged hepatectomies: results of a multicenter analysis. World J Surg 2014;38:1510-9.

4. Tanaka K, Matsuo K, Murakami T, et al. Associating liver partition and portal vein ligation for staged hepatectomy (ALPPS): short-term outcome, functional changes in the future liver remnant, and tumor growth activity. Eur J Surg Oncol 2015;41:506-12.

5. Schadde E, Raptis DA, Schnitzbauer AA, et al. Prediction of Mortality After ALPPS Stage-1: An Analysis of 320 Patients From the International ALPPS Registry. Ann Surg 2015;262:780-5; discussion 785-6.

6. Stavrou GA, Donati M, Fard-Aghaie MH, et al. Did the International ALPPS Meeting 2015 Have an Impact on Daily Practice? The Hamburg Barmbek Experience of 58 Cases. Visc Med 2017;33:456-61.

7. Li J, Girotti P, Königsrainer I, et al. ALPPS in right trisectionectomy: a safe procedure to avoid postoperative liver failure? J Gastrointest Surg 2013;17:956-61.

8. Linecker M, Björnsson B, Stavrou GA, et al. Risk Adjustment in ALPPS Is Associated With a Dramatic Decrease in Early Mortality and Morbidity. Ann Surg 2017;266:779-86.

9. Linecker M, Stavrou GA, Oldhafer KJ, et al. The ALPPS Risk Score: Avoiding Futile Use of ALPPS. Ann Surg 2016;264:763-71.

10. Eshmuminov D, Raptis DA, Linecker M, et al. Metaanalysis of associating liver partition with portal vein ligation and portal vein occlusion for two-stage hepatectomy. Br J Surg 2016;103:1768-82.

11. Codony C, López-Ben S, Albiol M, et al. Extreme liver surgery as treatment of liver tumors involving the hepatocaval confluence. Clin Transl Oncol 2016;18:1131-9.

12. Sargenti K, Prytz H, Nilsson E, et al. Predictors of mortality among patients with compensated and decompensated liver cirrhosis: the role of bacterial infections and infection-related acute-on-chronic liver failure. Scand J Gastroenterol 2015;50:875-83.

13. Khandoga A, Drefs $M$, Schoenberg $M$, et al. Differential significance of early surgical complications for acute and long-term recurrence-free survival following surgical resection of hepatocellular carcinoma: do comorbidities play a role? Eur J Gastroenterol Hepatol 2017;29:1045-53.

14. Chang CM, Yin WY, Su YC, et al. Preoperative risk score predicting 90-day mortality after liver resection in a population-based study. Medicine (Baltimore) 2014;93:e59.

15. Mehta HB, Dimou F, Adhikari D, et al. Comparison of Comorbidity Scores in Predicting Surgical Outcomes. Med Care 2016;54:180-7.

16. Charlson ME, Pompei P, Ales KL, et al. A new method of classifying prognostic comorbidity in longitudinal studies: development and validation. J Chronic Dis 1987;40:373-83.

17. Huiskens J, Schadde E, Lang H, et al. Avoiding postoperative mortality after ALPPS-development of a tumor-specific risk score for colorectal liver metastases. HPB (Oxford) 2019;21:898-905.

18. Linecker M, Kuemmerli C, Kambakamba P, et al. Performance validation of the ALPPS risk model. HPB (Oxford) 2019;21:711-21.

19. Mosteller RD. Simplified calculation of body-surface area. N Engl J Med 1987;317:1098.

20. Vauthey JN, Abdalla EK, Doherty DA, et al. Body surface area and body weight predict total liver volume in Western adults. Liver Transpl 2002;8:233-40.

21. Dindo D, Demartines N, Clavien PA. Classification of surgical complications: a new proposal with evaluation in a cohort of 6336 patients and results of a survey. Ann Surg 2004;240:205-13.

22. Schnitzbauer AA, Lang SA, Goessmann H, et al. Right portal vein ligation combined with in situ splitting induces rapid left lateral liver lobe hypertrophy enabling 2-staged extended right hepatic resection in small-for-size settings. Ann Surg 2012;255:405-14.

23. Alvarez FA, Ardiles V, Sanchez Claria R, et al. Associating liver partition and portal vein ligation for staged hepatectomy (ALPPS): tips and tricks. J Gastrointest Surg 2013;17:814-21.

24. Petrowsky H, Györi G, de Oliveira M, et al. Is partialALPPS safer than ALPPS? A single-center experience. Ann Surg 2015;261:e90-2.

25. Alvarez FA, Ardiles V, de Santibañes M, et al. Associating liver partition and portal vein ligation for staged hepatectomy offers high oncological feasibility with 
adequate patient safety: a prospective study at a single center. Ann Surg 2015;261:723-32.

26. Robles R, Parrilla P, López-Conesa A, et al. Tourniquet modification of the associating liver partition and portal ligation for staged hepatectomy procedure. Br J Surg 2014;101:1129-34; discussion 1134.

27. Gall TM, Sodergren MH, Frampton AE, et al. Radiofrequency-assisted Liver Partition with Portal vein ligation (RALPP) for liver regeneration. Ann Surg 2015;261:e45-6.

28. de Santibañes E, Alvarez FA, Ardiles V, et al. Inverting the ALPPS paradigm by minimizing first stage impact: the Mini-ALPPS technique. Langenbecks Arch Surg 2016;401:557-63.

29. Oldhafer KJ, Stavrou GA, van Gulik TM, et al. ALPPS-Where Do We Stand, Where Do We Go?: Eight Recommendations From the First International Expert Meeting. Ann Surg 2016;263:839-41.

30. Pang YY. The Brisbane 2000 terminology of liver anatomy and resections. HPB 2000; 2:333-39. HPB (Oxford) 2002;4:99; author reply 99-100.

31. Royston P, Altman DG, Sauerbrei W. Dichotomizing continuous predictors in multiple regression: a bad idea. Stat Med 2006;25:127-41.

32. Tripepi G, Jager KJ, Dekker FW, et al. Linear and logistic regression analysis. Kidney Int 2008;73:806-10.

33. Riley RD, Ensor J, Snell KIE, et al. Calculating the sample size required for developing a clinical prediction model. BMJ 2020;368:m441.

34. DeLong ER, DeLong DM, Clarke-Pearson DL. Comparing the areas under two or more correlated receiver operating characteristic curves: a nonparametric approach. Biometrics 1988;44:837-45.

35. Olthof PB, van Dam R, Jovine E, et al. Accuracy of estimated total liver volume formulas before liver resection. Surgery 2019;166:247-53.

36. D'Haese JG, Neumann J, Weniger M, et al. Should ALPPS be Used for Liver Resection in Intermediate-Stage HCC? Ann Surg Oncol 2016;23:1335-43.

37. Ribero D, Chun YS, Vauthey JN. Standardized liver volumetry for portal vein embolization. Semin Intervent Radiol 2008;25:104-9.

38. Urata K, Kawasaki S, Matsunami H, et al. Calculation of child and adult standard liver volume for liver transplantation. Hepatology 1995;21:1317-21.

39. Redlarski G, Palkowski A, Krawczuk M. Body surface area formulae: an alarming ambiguity. Sci Rep 2016;6:27966.

40. Nix DE, Mayersohn M, Erstad BL. Should estimates of glomerular filtration rate and creatinine clearance be indexed to body surface area for drug dosing? Am J Health Syst Pharm 2017;74:1814-9.

41. Hendriksen JM, Geersing GJ, Moons KG, et al. Diagnostic and prognostic prediction models. J Thromb Haemost 2013;11 Suppl 1:129-41.

42. Saha SK, Zhu AX, Fuchs CS, et al. Forty-Year Trends in Cholangiocarcinoma Incidence in the U.S.: Intrahepatic Disease on the Rise. Oncologist 2016;21:594-9.

43. Tanaka K. Modified ALPPS procedures: more safety through less invasive surgery. Langenbecks Arch Surg 2017;402:563-74.

44. Cai YL, Song PP, Tang W, et al. An updated systematic review of the evolution of ALPPS and evaluation of its advantages and disadvantages in accordance with current evidence. Medicine (Baltimore) 2016;95:e3941.

45. Linecker M, Kambakamba P, Reiner CS, et al. How much liver needs to be transected in ALPPS? A translational study investigating the concept of less invasiveness. Surgery 2017;161:453-64.

46. Peduzzi P, Concato J, Kemper E, et al. A simulation study of the number of events per variable in logistic regression analysis. J Clin Epidemiol 1996;49:1373-9.

47. Olthof PB, van Gulik TM, Bennink RJ. The HIBA Index for ALPPS, Preliminary Results to Interpret With Caution. Ann Surg 2018;267:e97-8.

48. Serenari M, Collaud C, Alvarez FA, et al. Interstage Assessment of Remnant Liver Function in ALPPS Using Hepatobiliary Scintigraphy: Prediction of Posthepatectomy Liver Failure and Introduction of the HIBA Index. Ann Surg 2018;267:1141-7.

49. Truant S, Baillet C, Deshorgue AC, et al. Contribution of hepatobiliary scintigraphy in assessing ALPPS most suited timing. Updates Surg 2017;69:411-9.

50. Olthof PB, Tomassini F, Huespe PE, et al. Hepatobiliary scintigraphy to evaluate liver function in associating liver partition and portal vein ligation for staged hepatectomy: Liver volume overestimates liver function. Surgery 2017;162:775-83.

51. Nadalin S, Capobianco I, Li J, et al. Indications and limits for associating liver partition and portal vein ligation for staged hepatectomy (ALPPS). Lessons Learned from 15 cases at a single centre. Z 
Gastroenterol 2014;52:35-42.

52. Cieslak KP, Olthof PB, van Lienden KP, et al. Assessment of Liver Function Using (99m)Tc-Mebrofenin

Cite this article as: Capobianco I, Oldhafer KJ, Fard-Aghaie MH, Robles-Campos R, Brusadin R, Petrowsky H, Linecker M, Mehrabi A, Hoffmann K, Li J, Heumann A, HernandezAlejandro R, Tun-Abraham ME, Jovine E, Serenari M, Bjornsson B, Sandström P, Alikhanov R, Efanov M, Muiesan P, Schlegel A, van Gulik TM, Olthof PB, Stavrou GA, SernaHiguita LM, Königsrainer A, Nadalin S. Development and internal validation of the Comprehensive ALPPS Preoperative Risk Assessment (CAPRA) score: is the patient suitable for Associating Liver Partition and Portal vein ligation for Staged hepatectomy (ALPPS)? HepatoBiliary Surg Nutr 2022;11(1):52-66. doi: 10.21037/hbsn-21-396
Hepatobiliary Scintigraphy in ALPPS (Associating Liver Partition and Portal Vein Ligation for Staged Hepatectomy). Case Rep Gastroenterol 2015;9:353-60. 
Table S1 The different conditions were evaluated as defined by Charlson in his original paper

Comorbidities definition

Cardiovascular disease

* Angina includes patients with chronic exertional angina, those with a coronary artery bypass graft, and those initially admitted with unstable angina

* Myocardial infarction includes patients with one or more definite or probable myocardial infarctions; these patients were hospitalized and had electrocardiographic and/or enzyme changes. Patients with electrocardiographic changes alone were not designated as having had an infarction

* Congestive heart failure includes patients who had exertional or paroxysmal nocturnal dyspnea and who responded symptomatically (or on physical examination) to digitalis, diuretics, or afterload-reducing agents. It does not include patients who are on medication, but have had no symptomatic response and no evidence of improvement of physical signs

* Arrhythmia includes patients with chronic atrial fibrillation or flutter $\{36 \%$ [33]\}, sick sinus syndrome, or ventricular arrhythmias requiring chronic treatment

* Valvular disease includes patients with hemodynamically significant aortic stenosis and/or insufficiency, those with significant mitral stenosis and/or insufficiency, and those with prosthetic aortic or mitral valves and those with symptomatic mitral valve prolapse asymmetric septal hypertrophy requiring treatment, or tricuspid insufficiency

- Peripheral vascular disease includes patients with intermittent claudication or those who had a bypass for arterial insufficiency those with gangrene or acute arterial insufficiency, and those with an untreated thoracic or abdominal aneurysm ( $6 \mathrm{~cm}$ or more)

* Hypertension includes patients with diastolic pressures over $120 \mathrm{mmHg}$; those with diastolic pressures between 100 and 120; and those with diastolic pressures below 100 as well as with controlled hypertension

\section{Neurological disease}

* Cerebrovascular disease includes patients with a history of a cerebrovascular accident with minor or no residual or transient ischemic attacks

Paralysis includes patents wit condition

* Dementia includes patients with chronic cognitive deficit

* Other neurologic conditions include patients with Parkinson's disease, uncontrolled seizures, or syncope without an identified cause or treatment

Pulmonary disease

* Mild pulmonary disease includes patients who are dyspneic with moderate activity without treatment or those who are dyspneic only with attacks (e.g., asthma)

* Moderate pulmonary disease includes patients who are dyspneic with slight activity, with or without treatment and those who are dyspneic with moderate activity despite treatment

* Severe pulmonary disease includes patients who are dyspneic at rest despite treatment, those who require constant oxygen, those with $\mathrm{CO}_{2}$ retention and those with a baseline $\mathrm{PO}_{2}$ below 50 tor

Endocrine disorders:

* Severe diabetes includes patients with retinopathy, neuropathy, or nephropathy

* Moderate diabetes includes patients who were previously hospitalized for ketoacidosis, hyperosmolar coma, or check-up and those with juvenile onset or brittle diabetes

* Mild diabetes includes all other diabetes treated with insulin or oral hypoglycemia, but not diet alone

* Other endocrine includes patients with hypopituitarism, adrenal insufficiency, and recurrent acidosis

Renal disease

* Severe renal disease includes patients on dialysis, those who had a transplant, and those with uremia

* Moderate renal insufficiency includes patients with serum creatinine of $>3 \mathrm{mg} \%$

* Mild renal includes those with serum creatinine of $2-3 \mathrm{mg} \%$

Liver disease

* Severe liver disease consists of patients with cirrhosis, portal hypertension and a history of variceal bleeding

* Moderate liver disease consists of cirrhosis with portal hypertension, but without bleeding

* Mild liver disease consists of cirrhosis without portal hypertension or chronic hepatitis

Gastrointestinal disease

* Inflammatory bowel disease includes patients with ulcerative colitis or regional enteritis

* Peptic ulcer disease includes patients who required treatment for an ulcer, including a bleeding ulcer

* Gastrointestinal bleeding includes those who have had bleeding requiring transfusion for causes other than ulcer

Immunological, hematological and rheumatological diseases

* Acquired immune deficiency syndrome includes patients with definite or probable AIDS, i.e., AIDS-related complex

* Lymphoma includes patients with Hodgkin's, Iymphosarcoma, Waldenstrom's macroglobulinemia, myeloma, and other lymphomas

* Leukemia includes patients with acute or chronic myelogenous leukemia, acute or chronic lymphocytic leukemia, and polycythemia vera

* Rheumatologic disease includes patients with systemic lupus erythematous, polymyositis, mixed connective tissue disease polymyalgia rheumatica, and moderate to severe rheumatoid arthritis

* Coagulopathy includes patients with a circulating anticoagulant or other coagulopathy Tumors

* Tumor designates patients with solid tumors without documented metastases, but initially treated in the last five years, including liver, breast, colon, lung, and a variety of other tumors

* Metastatic cancer includes patients with metastatic solid tumors, including colon, breast, lung, and other tumors

Charlson's original paper: Charlson ME, Pompei P, Ales KL, et al. A new method of classifying prognostic comorbidity in longitudinal studies: development and validation. J Chronic Dis 1987;40:373-83. 
Table S2 Univariate analysis for postoperative mortality

\begin{tabular}{lcccc}
\hline Characteristic & Regression coefficient & OR & $95 \% \mathrm{Cl}$ & $\mathrm{P}$ \\
\hline Age & 0.091 & 1.095 & $1.06-1.131$ & $<0.001$ \\
Sex, male & 0.226 & 1.254 & $0.706-2.227$ & 0.441 \\
BMI & -0.33 & 0.967 & $0.906-1.033$ & 0.320 \\
BSA & -1.294 & 0.274 & $0.081-0.934$ & 0.038 \\
SLV & -0.001 & 0.999 & $0.998-1.000$ & 0.038
\end{tabular}

CRLM and OM

1

Biliary

$\mathrm{HCC}$ and OP

Primary tumor

Surgery

Classic ALPPS vs. modified

Open approach

Surgical approach of the hepatic hilus vs. PVE

(hybrid + RALPPS + tourniquet)

Parenchyma surgical vs. other

Classic ALPPS (vs. partial)

Hybrid ALPPS

Tourniquet ALPPS

RALPPS

Resection of more than 4 segments

1.398

0.927

1.204

4.046

2.526
3.333

2.258-7.248

$1.099-5.806$

$1.943-5.718$

1.906

0.645

19.457

1.757

0.944

$-1.166$

$-0.457$

$-0.77$

0.619

Comorbidities

Presence of comorbidities

Number of comorbidities

$\mathrm{CC}$

$\mathrm{aCCl}$

Cardiovascular disease

Angina

Myocardial infarction

Congestive heart failure

Arrhythmia

Valvular disease

Peripheral vascular disease

Hypertension

Severe cardiovascular disease

Neurological disease

Cerebrovascular disease

Paralysis

Dementia

Other neurological disease

Pulmonary disease

Severe pulmonary disease

Moderate pulmonary disease

Mild pulmonary disease

COPD

Endocrine disorders

Moderate and severe diabetes

Severe diabetes

Moderate diabetes

Mild diabetes

Other endocrine disease

Renal disease

Severe renal disease

Moderate renal disease

Mild renal disease

Liver disease

Severe liver disease

Moderate liver disease

Mild liver disease

Gastrointestinal disease

Inflammatory bowel disease

Peptic ulcer

Gastrointestinal bleeding

Immunological or hematological disease

AIDS

\section{Lymphoma}

Leukemia

Rheumatologic disease

Coagulopathy

Any tumor

Any non-metastatic tumor

Metastatic solid tumor

The table displays the respective logistic regression coefficients and OR with $95 \% \mathrm{Cl}$ for pre-Stage 1 variables. ${ }^{1}$, severe cardiovascular disease: any of congestive heart disease, myocardial infarction or peripheral vascular disease, $\mathrm{OR}$, odds ratios: $\mathrm{Cl}$ confidence intervals; BMI, body mass index: BSA, body surface index according to the Mosteller formula: SLV standard liver volume according to the Vauthey formula: CRLM, colorectal liver metastasis: OM, other metastatic liver tumor: HCC, hepatocellular carcinoma: OP, other primary liver formula, CRLM, colorectal liver metastasis, OM, other metastatic liver tumor, HCC, hepatocellular carcinoma, OP, other primary liver radiofrequency-assisted ALPPS; aCCI, age-adjusted Charlson Comorbidity Index; CCl, Charlson Comorbidity Index.

$1.005-21.035 \quad 0.049$

$0.895-6.329 \quad 0.082$

$0.090-5.971 \quad 0.773$

$1.982-16.232 \quad 0.001$

$1.096-3.158 \quad 0.021$

2.109-8.265 $<0.00$

$0.382-4.986 \quad 0.622$

$0.254-5.546 \quad 0.827$

$0.163-13.494$

$0.510-2.838 \quad 0.672$

1.000

$0.136-10.304 \quad 0.878$

$0.212-2.483 \quad 0.610$

0.335-4.232 0.788

$\begin{array}{ll}1.283-4.122 & 0.005\end{array}$

$1.455-12.339 \quad 0.008$

$\begin{array}{ll}1.514-56.428 & 0.016\end{array}$

1.004-12.424 $\quad 0.049$

$1.169-4.702 \quad 0.016$

$0.408-2.973 \quad 0.097$
$2.465-45.435$

2.465-45.435 0.002

0.999

$1.514-56.428 \quad 0.016$

$\begin{array}{ll}0.267-3.235 & 0.909\end{array}$

$\begin{array}{ll}0.102-6.955 & 0.873\end{array}$

$0.215-4.502 \quad 0.984$

$\begin{array}{ll}0.445-6.048 & 0.457\end{array}$

1.086-136.081 0.043

$\begin{array}{ll}1.254-5.546 & 0.827\end{array}$

$0.635-6.507 \quad 0.232$

- $\quad-$

$0.000 \quad 1.000$

$1.004-12.424-0.049$

$0.000 \quad 1.000$

$\begin{array}{llll}-0.537 & 0.584 & 0.074-4.643 & 0.611 \\ -0.537 & 0.584 & 0.074-4.643 & 0.611\end{array}$

$0.074-4.643$

$\begin{array}{llll}-0.537 & 0.584 & 0.074-4.643 & 0.611\end{array}$

